\title{
Untersuchungen zum Einfluss des Plattenaufbaus auf \\ ausgewählte Eigenschaften von Massivholzplatten
}

\author{
Journal Article \\ Author(s): \\ Bader, Hervé; Niemz, Peter; Sonderegger, Walter \\ Publication date: \\ 2007 \\ Permanent link: \\ https://doi.org/10.3929/ethz-b-000005134
}

Rights / license:

In Copyright - Non-Commercial Use Permitted

Originally published in:

European Journal of Wood and Wood Products 65(3), https://doi.org/10.1007/s00107-006-0143-7 


\title{
Untersuchungen zum Einfluss des Plattenaufbaus auf ausgewählte Eigenschaften von Massivholzplatten
}

\author{
Hervé Bader · Peter Niemz • Walter Sonderegger
}

Published online: 19 September 2006

(C) Springer-Verlag 2006

\begin{abstract}
Zusammenfassung An labortechnisch hergestellten Massivholzplatten aus Fichtenholz wurden die Wärmeleitfähigkeit, die Gleichgewichtsfeuchte, die Längenquellung und der Wasserdampfdiffusionswiderstand bestimmt. Die Wärmeleitfähigkeit liegt im Normalklima im Bereich von 0,09-0,11 W/m · K. Hohlräume in der Mittellage (Schlitze, Abstände zwischen den einzelnen Brettlamellen) führen zu einer geringen Reduzierung der Wärmeleitfähigkeit. Auch die Anordnung der Jahrringe wirkt sich aus. Auf den Wasserdampfdiffusionswiderstand haben die Orientierung der Jahrringe in den Lagen und die Hohlräume in den Mittellagen einen Einfluss. Die Gleichgewichtsfeuchte liegt oberhalb von $35 \%$ rel. Luftfeuchte etwas unterhalb derjenigen von Fichtenholz. Bei Holzwerkstoffmittellagen ist sie durch die geringere Ausgleichsfeuchte der Holzpartikelwerkstoffe geringer als bei reinen Vollholzmittellagen.
\end{abstract}

\section{Investigation on the influence of the panel composition on selected properties of three-layer solid wood panels}

\begin{abstract}
The coefficient of thermal conductivity, the equilibrium moisture content, the in-plane swelling and the water vapour resistance of laboratory-produced three-layer solid wood panels of Norway spruce wood were tested. The coefficient of thermal conductivity is in the range of $0.09-0.11 \mathrm{~W} / \mathrm{m} \cdot \mathrm{K}$ in normal climate. The voids in the middle layer (slots, spaces between the lamellas) cause a slight decrease of the coefficient of thermal conductivity. The orientation of the growth rings has an effect on the thermal conductivity as well. The water vapour resistance is affected
\end{abstract}

H. Bader · P. Niemz ( $)$. W. Sonderegger

Institut für Baustoffe, Holzphysik, ETH Zürich,

Schafmattstrasse 6,

8093 Zürich, Switzerland

e-mail: niemzp@ethz.ch by the orientation of the growth rings in the layers and the voids in the middle layer. The equilibrium moisture content above a relative humidity of $35 \%$ is slightly below the one of solid spruce wood. The equilibrium moisture content of solid wood panels with a middle layer of wood-based material is smaller because of its lower equilibrium moisture content compared with solid wood.

\section{Einleitung}

Im Bauwesen werden verstärkt mehrschichtige Massivholzplatten für konstruktive Zwecke eingesetzt. Dabei werden neben konventionellen Mehrschichtplatten auch solche mit Mittellagen aus Holzwerkstoffen verwendet (Tobisch und Krug 2002a, 2002b). Zunehmend wird versucht, den Plattenaufbau zu optimieren, um Kosten einzusparen, und auch, um die durch die Querverleimung entstehenden Spannungen zu vermindern. So werden die Mittellagen geschlitzt, Decklagen mit Riftschnitt eingesetzt oder auch Holzwerkstoffe wie Spanplatten und OSB in der Mittellage verwendet. Bisher gibt es vergleichsweise wenige Arbeiten zu den Beziehungen zwischen Plattenaufbau und Platteneigenschaften. Dies betrifft insbesondere die Wärmeleitfähigkeit, den Diffusionswiderstand, das Sorptionsverhalten und die Quellung, aber auch die Formbeständigkeit und die Rissbildung im Differenzklima. Im Rahmen der vorliegenden Arbeit wurden an labortechnisch hergestellten Platten mit variablem Aufbau ausgewählte Eigenschaften getestet.

\section{Erkenntnisstand}

$\mathrm{Zu}$ mechanischen Eigenschaften von Massivholzplatten und zum Einfluss des Plattenaufbaus liegen vergleichsweise viele Arbeiten vor. So wurden von Kreuzinger (1991), Blaß und Fellmoser (2003) sowie Donzé und Niemz (2004) Untersu- 
chungen zur Berechnung der mechanischen Eigenschaften von Massivholzplatten publiziert. In der Praxis erfolgt die Berechnung noch überwiegend in Anlehnung an die Berechnungsgrundlagen von Baufurniersperrholz (DIN 68705-5 Beiblatt 1). Experimentell wurde der Einfluss des Plattenaufbaus u.a. von Krug (1999), Tobisch und Krug (2002a, 2002b, 2002c) sowie Steiger und Niemz (2004) untersucht. Tobisch und Krug (2002a, 2002b) prüften auch die Kombination von Decklagen aus Vollholz und Holzwerkstoffmittellagen. Für die Wärmeleitfähigkeit $(\lambda)$ liegen nur Literaturangaben aus Firmenschriften vor. Es wird meist ein Wert für $\lambda$ von $0,12-0,14 \mathrm{~W} / \mathrm{m} \cdot \mathrm{K}$ angegeben. Die Werte für den Wasserdampfdiffusionswiderstand $(\mu)$ schwanken sowohl in Firmenschriften als auch in wissenschaftlichen Publikationen deutlich. So wird in Merz et al. (1997) ein Wert für $\mu$ von 20-160 angegeben, Radovic et al. (2001) geben 50 (feucht) und 400 (trocken) an. Die Gleichgewichtsfeuchte von Massivholzplatten aus Fichtenholz liegt leicht unter der von Fichtenvollholz. Für die differenzielle Längenquellung wurden in Abhängigkeit vom Plattenaufbau Werte von 0,016\%-0,045\%/\% ermittelt (Popper et al. 2004). Insgesamt streuen die publizierten Ergebnisse in einem weiten Bereich.

\section{Versuchsmaterial und Methodik}

\subsection{Versuchsmaterial}

\section{Als Versuchsmaterial dienten}

- industriell verklebte Lagen aus Fichtenholz (Picea abies [L.] Karst.) für Massivholzplatten (Dicken: 10, 14, 20,
30, $40 \mathrm{~mm}$; teilweise wechselseitig von oben und unten eingesägt, um Spannungsspitzen abzubauen)

- MDF- und OSB-Platten als Mittellagen

Die Lagen wurden vor der Verklebung im Normalklima bei $20^{\circ} \mathrm{C}$ und $65 \%$ rel. Luftfeuchte klimatisiert. Die Verklebung erfolgte mit dem 1-Komponenten Polyurethanklebstoff PURBOND HB 181. Die Leimauftragsmenge betrug $180 \mathrm{~g} / \mathrm{m}^{2}$, die Presszeit $6,5 \mathrm{~h}$, der Pressdruck 0,7 bar. Von der Oberfläche der OSB-Platten wurde vor der Verklebung beidseitig je $1 \mathrm{~mm}$ abgeschliffen, um die Presshaut zu beseitigen. Tabelle 1 zeigt die Versuchsübersicht. Je Variante wurden 6 Platten im Format $500 \mathrm{~mm} \times 500 \mathrm{~mm}$ hergestellt: 3 Platten zur Messung der Wärmeleitfähigkeit und 3 Platten zur Herstellung der Proben für die Bestimmung des Diffusionswiderstandes und der Längenquellung.

\subsection{Versuchsdurchführung}

\subsubsection{Wärmeleitfähigkeit}

Je Variante wurde an 3 Platten die Wärmeleitfähigkeit mit dem Wärmeleitfähigkeitsmessgerät , $\lambda$-Meter EP 500“ gemessen. Die Messungen erfolgten bei $10^{\circ} \mathrm{C}, 25^{\circ} \mathrm{C}$ und $40^{\circ} \mathrm{C}$. Daraus wurde dann durch Extrapolation der Wert bei $10^{\circ} \mathrm{C}$ bestimmt. Die Prüfung erfolgte nach DIN 52612 Teil 1. Vor der Prüfung wurden die Proben im Normalklima bei $20^{\circ} \mathrm{C}$ und $65 \%$ rel. Luftfeuchte klimatisiert. Zur besseren Vergleichbarkeit wurden die Ergebnisse der Messungen an den Platten aus reinen Holzlagen auf eine einheitliche, mittlere Rohdichte von $437 \mathrm{~kg} / \mathrm{m}^{3}$ und eine einheitliche Holzfeuchte von $12 \%$ umgerechnet (Tabelle 2).
Tabelle 1 Versuchsaufbau Table 1 Experimental setup
$\mathrm{G}=$ geschlitzt (slotted);

st $=$ stehende Jahrringe (vertical growth rings); $1=$ liegende Jahrringe (horizontal growth rings); 0, 5, 10, $30=$ Abstand zwischen den Lamellen (space between the lamellas)

\begin{tabular}{|c|c|c|c|}
\hline Nr. & $\begin{array}{l}\text { Plattenaufbau } \\
\text { (Schichtdicken } \\
\text { in } \mathrm{mm} \text { ) }\end{array}$ & $\begin{array}{c}\text { Lamellen- } \\
\text { breite } \\
\text { (Mittellage) } \\
\end{array}$ & $\begin{array}{c}\text { Lamellen- } \\
\text { breite } \\
\text { (Decklagen) } \\
\end{array}$ \\
\hline 1 & $10 / 10 / 10$ & $70 \mathrm{~mm}$, verleimt & $70 \mathrm{~mm}$, verleimt \\
\hline 2 & $10 / 20 / 10$ & $70 \mathrm{~mm}$, verleimt & $70 \mathrm{~mm}$, verleimt \\
\hline 3 & $10 / 40 / 10$ & $70 \mathrm{~mm}$, verleimt & $70 \mathrm{~mm}$, verleimt \\
\hline 4 & $7 / 14_{\mathrm{G}} / 7$ & $\begin{array}{l}50-80 \mathrm{~mm} \text {, verleimt, doppelseitig geschlitzt } \\
(11 \mathrm{~mm} \text { tief, im Abstand von } 10 \mathrm{~mm} \text { alternierend) }\end{array}$ & $100 \mathrm{~mm}$, verleimt \\
\hline 5 & $20 / 30_{\mathrm{G}} / 20$ & $\begin{array}{c}70 \mathrm{~mm} \text {, verleimt, doppelseitig, geschlitzt } \\
(20 \mathrm{~mm} \text { tief, im Abstand von } 16 \mathrm{~mm} \text { alternierend) }\end{array}$ & $70 \mathrm{~mm}$, verleimt \\
\hline 6 & $10_{\mathrm{st}} / 10 / 10_{\mathrm{st}}$ & $70 \mathrm{~mm}$, verleimt & $\begin{array}{l}70 \mathrm{~mm} \text {, verleimt, } \\
\text { stehende Jahrringe }\end{array}$ \\
\hline 7 & $14_{\mathrm{st}} / 14_{\mathrm{st}} / 14_{\mathrm{st}}$ & $25 \mathrm{~mm}$, verleimt, stehende Jahrringe & $\begin{array}{l}25 \mathrm{~mm} \text {, verleimt, } \\
\text { stehende Jahrringe }\end{array}$ \\
\hline 8 & $10 / 19_{\mathrm{MDF}} / 10$ & MDF-Platte & $70 \mathrm{~mm}$, verleimt \\
\hline 9 & $10 / 17_{\mathrm{OSB}} / 10$ & OSB-Platte & $70 \mathrm{~mm}$, verleimt \\
\hline 10 & $10 / 10_{0} / 10$ & $70 \mathrm{~mm}$, unverleimt & $70 \mathrm{~mm}$, verleimt \\
\hline 11 & $10 / 10_{5} / 10$ & $70 \mathrm{~mm}$, mit $5 \mathrm{~mm}$ Abstand & $70 \mathrm{~mm}$, verleimt \\
\hline 12 & $10 / 10_{10} / 10$ & $70 \mathrm{~mm}$, mit $10 \mathrm{~mm}$ Abstand & $70 \mathrm{~mm}$, verleimt \\
\hline 13 & $10 / 10_{30} / 10$ & $70 \mathrm{~mm}$, mit $30 \mathrm{~mm}$ Abstand & $70 \mathrm{~mm}$, verleimt \\
\hline 14 & $10_{1} / 10 / 10_{1}$ & $70 \mathrm{~mm}$, verleimt & $\begin{array}{l}70 \mathrm{~mm} \text {, verleimt, } \\
\text { liegende Jahrringe }\end{array}$ \\
\hline
\end{tabular}


Tabelle 2 Wärmeleitfähigkeit der geprüften Varianten

Table 2 Coefficients of thermal conductivity of the divers board types
$T_{c}=$ Temperaturkoeffizient $=$ Zunahme von $\lambda$ je $1{ }^{\circ} \mathrm{C}$ $T_{c}=$ Coefficient of temperature $=$ increase of $\lambda$ per $1{ }^{\circ} \mathrm{C}$

\begin{tabular}{|c|c|c|c|c|c|c|c|}
\hline \multirow[t]{2}{*}{$\begin{array}{l}\text { Variante } \\
\end{array}$} & \multirow{2}{*}{$\begin{array}{l}\text { Plattenaufbau } \\
\text { [mm] }\end{array}$} & \multirow{2}{*}{$\begin{array}{l}\text { Rohdichte } \\
{\left[\mathrm{kg} / \mathrm{m}^{3}\right]}\end{array}$} & \multirow{2}{*}{$\begin{array}{c}\text { Holzfeuchte } \\
{[\%]}\end{array}$} & \multicolumn{3}{|c|}{ Wärmeleitfähigkeit } & \multirow[b]{2}{*}{$\begin{array}{c}\text { Temperatur- } \\
\text { einfluss } \\
T_{c} \\
{\left[\mathrm{~W} / \mathrm{m} \cdot \mathrm{K}^{2}\right]} \\
\cdot 10^{-3}\end{array}$} \\
\hline & & & & $\begin{array}{c}\lambda-10 \\
{[\mathrm{~W} / \mathrm{m} \cdot \mathrm{K}]}\end{array}$ & $\begin{array}{c}\lambda-10 \\
(\mathrm{u}=12 \%) \\
{[\mathrm{W} / \mathrm{m} \cdot \mathrm{K}]}\end{array}$ & $\begin{array}{c}\lambda-10 \\
\left(\rho=437 \mathrm{~kg} / \mathrm{m}^{3}\right) \\
{[\mathrm{W} / \mathrm{m} \cdot \mathrm{K}]}\end{array}$ & \\
\hline 1 & $10 / 10 / 10$ & 417 & 11,65 & 0,091 & 0,092 & 0,096 & 0,28 \\
\hline 2 & $10 / 20 / 10$ & 436 & 12,27 & 0,096 & 0,096 & 0,096 & 0,24 \\
\hline 3 & $10 / 40 / 10$ & 432 & 12,58 & 0,099 & 0,098 & 0,099 & 0,43 \\
\hline 4 & $7 / 14_{\mathrm{G}} / 7$ & 435 & 11,55 & 0,095 & 0,096 & - & 0,30 \\
\hline 5 & $20 / 30_{\mathrm{G}} / 20$ & 394 & 12,59 & 0,094 & 0,093 & - & 0,48 \\
\hline 6 & $10_{\mathrm{st}} / 10 / 10_{\mathrm{st}}$ & 424 & 11,57 & 0,088 & 0,088 & 0,091 & 0,22 \\
\hline 7 & $14_{\mathrm{st}} / 14_{\mathrm{st}} / 14_{\mathrm{st}}$ & 467 & 10,78 & 0,104 & 0,106 & 0,100 & 0,26 \\
\hline 8 & $10 / 19_{\mathrm{MDF}} / 10$ & 596 & 9,20 & 0,105 & - & - & 0,30 \\
\hline 9 & $10 / 17_{\mathrm{OSB}} / 10$ & 544 & 10,47 & 0,107 & - & - & 0,27 \\
\hline 10 & $10 / 10_{0} / 10$ & 446 & 12,00 & 0,101 & 0,101 & 0,099 & 0,29 \\
\hline 11 & $10 / 10_{5} / 10$ & 443 & 12,56 & 0,100 & 0,100 & - & 0,31 \\
\hline 12 & $10 / 10_{10} / 10$ & 418 & 12,39 & 0,094 & 0,094 & - & 0,30 \\
\hline 13 & $10 / 10_{30} / 10$ & 397 & 11,67 & 0,091 & 0,091 & - & 0,38 \\
\hline 14 & $10_{1} / 10 / 10_{1}$ & 441 & 12,03 & 0,096 & 0,096 & 0,095 & 0,26 \\
\hline
\end{tabular}

Für die Feuchte- und Dichtekorrektur wurden die von Kollmann (1951) in Niemz (1993) angegebenen Formeln verwendet (Gl. 1 und 2):

Für den Einfluss der Holzfeuchte gilt:

$\lambda_{2}=\lambda_{1}\left[1-0,0125\left(u_{1}-u_{2}\right)\right]$,

$\lambda_{1}, \lambda_{2}=$ Wärmeleitfähigkeit bei Holzfeuchte $u_{1}$ bzw. $u_{2}$ $[\mathrm{W} / \mathrm{m} \cdot \mathrm{K}]$

$u_{1}, u_{2}=$ Holzfeuchten im Zustand 1 bzw. 2 [\%].

Für den Einfluss der Rohdichte gilt:

$\lambda_{\perp, 1}=0,026+0,195 \cdot \rho_{1} \cdot 10^{-3}$.

Daraus lässt sich folgende Gleichung zur Dichtekorrektur der Wärmeleitfähigkeit herleiten:

$\lambda_{\perp, 2}=\lambda_{\perp, 1}+0,195 \cdot\left(\rho_{2}-\rho_{1}\right) \cdot 10^{-3}$,

$\lambda_{\perp, 1}, \lambda_{\perp, 2}=$ Wärmeleitfähigkeit senkrecht zur Faserrichtung bei Rohdichte 1 bzw. $2[\mathrm{~W} /(\mathrm{m} \cdot \mathrm{K})]$

$\rho_{1}, \rho_{2}=$ Rohdichten 1 bzw. $2\left[\mathrm{~kg} / \mathrm{m}^{3}\right]$.

\subsubsection{Gleichgewichtsfeuchte und Längenquellung}

Die Längenquellung wurde nach DIN 52184, die Gleichgewichtsfeuchte in Anlehnung an DIN 52183 bestimmt.

Die Messung erfolgte an je 20 Proben parallel und auBer bei den Varianten 11-13 (s. Tabelle 1) auch senkrecht zur Faserrichtung der Decklagen. Das Probenformat betrug $20 \mathrm{~mm}$ ( Breite) $\times 200 \mathrm{~mm}$ ( Länge) $\times$ Plattendicke. Gemessen wurden in beiden Richtungen die faserparallelen Schichten, um den Randeinfluss gering zu halten (parallel zur Faserrichtung $\rightarrow$ Messung der Decklage; senkrecht zur Faserrichtung $\rightarrow$ Messung der Mittellage). Die Sorptions-Messungen erfolgten im Klima bei $20^{\circ} \mathrm{C}$ und $35 \%, 50 \%, 65 \%, 80 \%, 88 \%$ und $95 \%$ rel. Luftfeuchte, die Längenmessungen im Klima bei $20^{\circ} \mathrm{C}$ und $35 \%, 50 \%$, $65 \%$ und $80 \%$ rel. Luftfeuchte, da sich die Proben bei höheren Feuchten krümmten und dadurch das Messresultat verfälschten. Für die Längenmessung wurde die in Abb. 1 dargestellte Versuchsvorrichtung verwendet.

Aus den ermittelten Längenänderungen wurde das lineare Quellmaß $\alpha$ und die differenzielle Längenänderung $q$ (berechnet als Regression zwischen 35\% und 80\% rel. Luft-

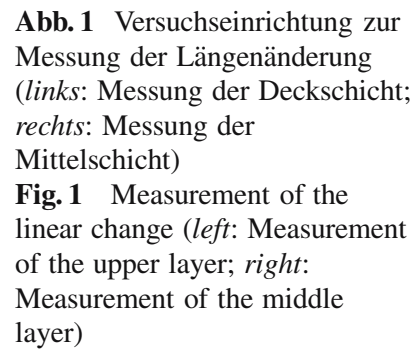

Abb. 1 Versuchseinrichtung zur Messung der Längenänderung (links: Messung der Deckschicht; rechts: Messung der Mittelschicht)

Fig. 1 Measurement of the linear change (left: Measurement the upper layer, right: layer)

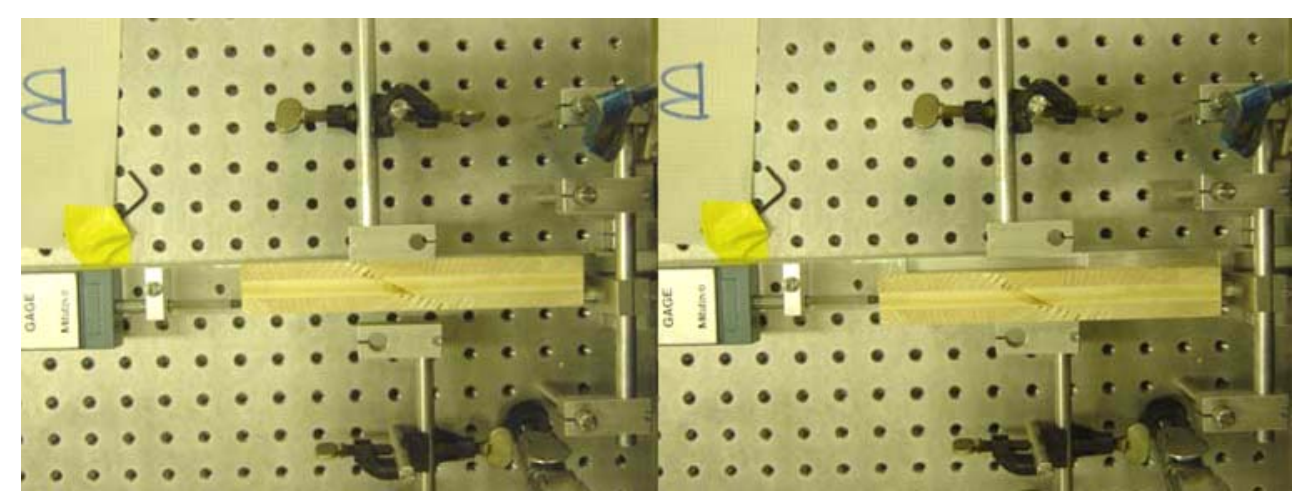


Abb. 2 Versuchsaufbau zur Messung des Wasserdampfdiffusionswiderstandes

Fig. 2 Experimental setup for the measurement of the water vapour resistance

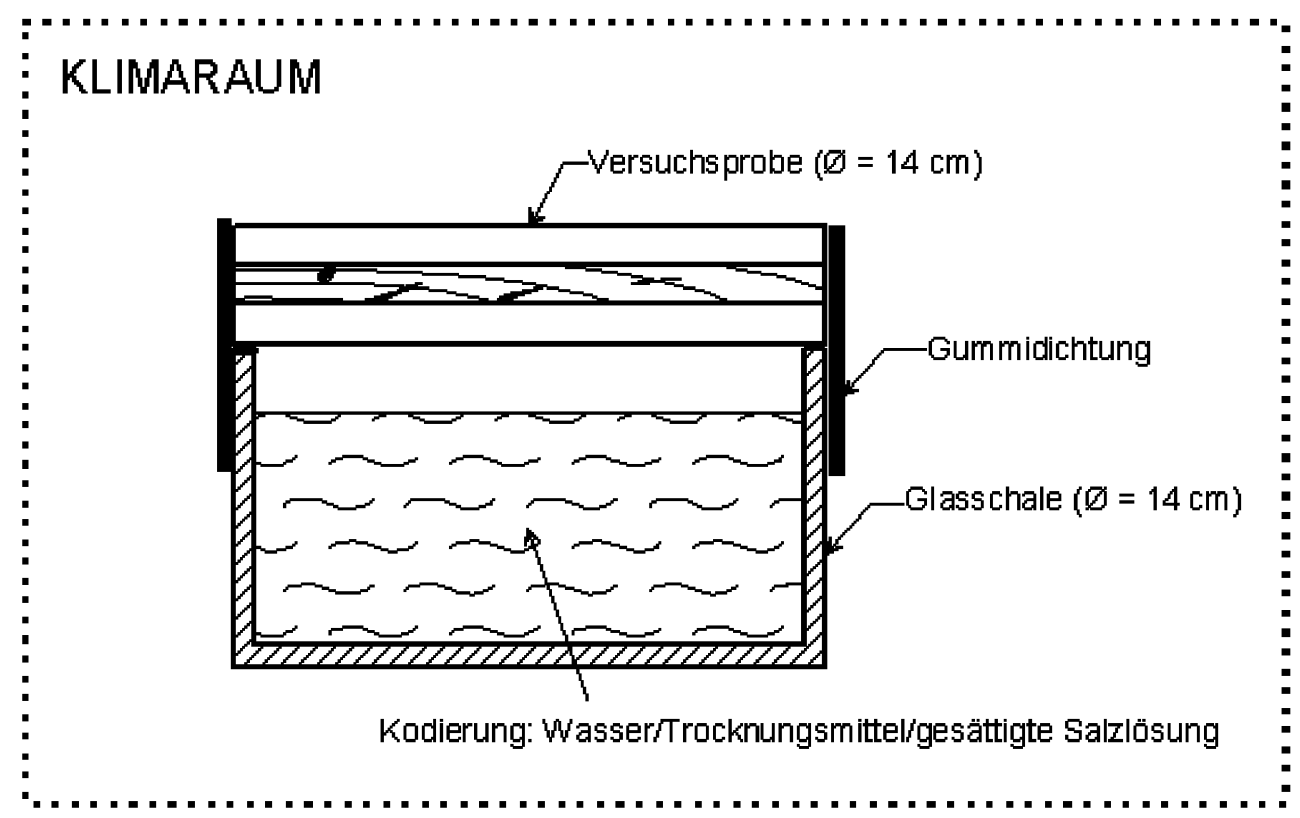

feuchte) analog den Gleichungen 4 und 5 bestimmt. Dabei wurde infolge Rissbildung bei der Darrtrocknung in beiden Gleichungen an Stelle von $l_{0}$ die Länge bei $35 \%$ rel. Luftfeuchte verwendet.

$\alpha=\frac{l_{2}-l_{1}}{l_{0}} \cdot 100$

$q=\frac{l_{F}-l_{T}}{l_{0} \cdot\left(u_{F}-u_{T}\right)} \cdot 100$,

$\alpha \quad=$ Lineares Quellmaß [\%]

$\mathrm{q}=$ Differenzielle Längenänderung [\%/\%]

$l_{0}=$ Länge der darrtrockenen Probe (hier: bei $35 \%$ rel. Luftfeuchte [mm])

$l_{1}, l_{2}=$ Länge der Probe bei der Holzfeuchte $u_{1}$ bzw. $u_{2}$ [mm]

$l_{F}=$ Länge der Probe im Feuchtklima (hier: bei $80 \%$ rel. Luftfeuchte $[\mathrm{mm}])$

$l_{T} \quad=$ Länge der Probe im Trockenklima (hier: bei $35 \%$ rel. Luftfeuchte $[\mathrm{mm}])$

$u_{F} \quad=$ Holzfeuchte im Feuchtklima [\%]

$u_{T} \quad=$ Holzfeuchte im Trockenklima [\%].

\subsubsection{Wasserdampfdiffusionswiderstand}

Der Wasserdampfdiffusionswiderstand wurde nach EN ISO 12572 (2001) bestimmt. Es wurde die in Abb. 2 dargestellte Versuchseinrichtung verwendet. Es wurde ein Differenzklima von $20{ }^{\circ} \mathrm{C} / 65 \%$ rel. Luftfeuchte und $20^{\circ} \mathrm{C} / 0 \%$ rel. Luftfeuchte (Silikagel) verwendet. Berechnet wurden die Wasserdampfdiffusionswiderstandszahl $\mu$ und die wasserdampfdiffusionsäquivalente Luftschichtdicke $s_{d}$. Je Variante wurden 3 Proben geprüft.

\section{Versuchsergebnisse}

\subsection{Allgemeines}

In den Tabellen zur Wärmeleitfähigkeit und zum Wasserdampfdiffusionswiderstand (Tabellen 2 und 5) wurden jeweils die Mittelwerte aus den 3 Messungen pro Variante angegeben, bei den Holzfeuchten der SorptionsMessungen (Tabelle 3) wurden ebenfalls nur die Mittelwerte aufgeführt (sehr geringe Streuung der Werte mit einem Variationskoeffizienten von $<3 \%$ ), in den Abbil-

Tabelle 3 Gleichgewichtsfeuchten bei verschiedenen relativen Luftfeuchten

Table 3 Moisture content at different relative humidities

\begin{tabular}{|c|c|c|c|c|c|c|c|}
\hline \multirow[t]{2}{*}{ Var.Nr. } & \multirow{2}{*}{$\begin{array}{l}\text { Platten- } \\
\text { aufbau }\end{array}$} & \multicolumn{6}{|c|}{ Holzfeuchte [\%] bei den rel. Luftfeuchten } \\
\hline & & $35 \%$ & $50 \%$ & $65 \%$ & $80 \%$ & $88 \%$ & $95 \%$ \\
\hline $\begin{array}{l}\text { Fichte } \\
\text { holz }\end{array}$ & & 8,5 & 11,1 & 12,5 & 16,8 & & 27,7 \\
\hline 1 & $10 / 1$ & 9,4 & 10,72 & 11,60 & 14,65 & 17,' & 25,5 \\
\hline 2 & & 7 & & 11,9 & 14 , & & 24,2 \\
\hline 3 & $10 / 40 / 10$ & 9,95 & 11,40 & 12,39 & 15,61 & & 26,31 \\
\hline 4 & $114_{\mathrm{G}} / 7$ & 9,13 & 10,27 & 11,27 & 15,14 & 17, & 24,25 \\
\hline 5 & $20 / 30_{\mathrm{G}} / 20$ & 9,92 & 11,36 & 12,40 & 15,39 & 18 , & 24,45 \\
\hline 6 & ${ }_{\mathrm{t}} / 10 / 10_{\mathrm{st}}$ & 9,72 & 11,13 & 12,07 & 15,00 & 18, & 26,48 \\
\hline 7 & ${ }_{\text {st }} / 14_{\text {st }} / 14_{\text {st }}$ & 9,35 & 10,43 & 11,20 & 14,60 & & 24,91 \\
\hline 8 & $19_{\mathrm{MDF}} / 10$ & 7,83 & & 9,32 & 12,32 & & 24,19 \\
\hline 9 & $/ 10$ & 8,55 & & 10,27 & 13 , & & 24,55 \\
\hline () & 10 & 9,70 & & 11,97 & 15 , & & 25,58 \\
\hline 1 & 0 & 65 & & 11,85 & 15 , & & 25,82 \\
\hline 12 & $/ 10$ & 9,62 & 10,97 & 11,85 & 15, & & 26,32 \\
\hline 13 & & 9,64 & 11,00 & 11,89 & 15,30 & 17, & 25,50 \\
\hline 14 & $10_{1} / 10 / 10_{1}$ & 9,93 & 11,07 & 12,00 & 15,01 & 17,99 & 24,37 \\
\hline
\end{tabular}

${ }^{1}$ Extrapoliert aus den Werten bei $80 \%$ und $95 \%$ relativer Luftfeuchte.

${ }^{1}$ Extrapolated from the values at a relative humidity of $80 \%$ and $95 \%$. 
Abb. 3 Einfluss der Rohdichte auf die Wärmeleitfähigkeit (Messwerte bei $25^{\circ} \mathrm{C}$ und $65 \%$ Luftfeuchte) und Vergleich mit den Werten von Kollmann (lineare Regression (Gl. 2) aus Messungen bei $27^{\circ} \mathrm{C}$ und $12 \%$ Holzfeuchte)

Fig. 3 Influence of the density on the thermal conductivity (values at $25^{\circ} \mathrm{C}$ and a relative humidity of $65 \%$ ) compared with values from Kollmann (linear regression (Eq. 2) of values at $27^{\circ} \mathrm{C}$ and $12 \%$ moisture content)

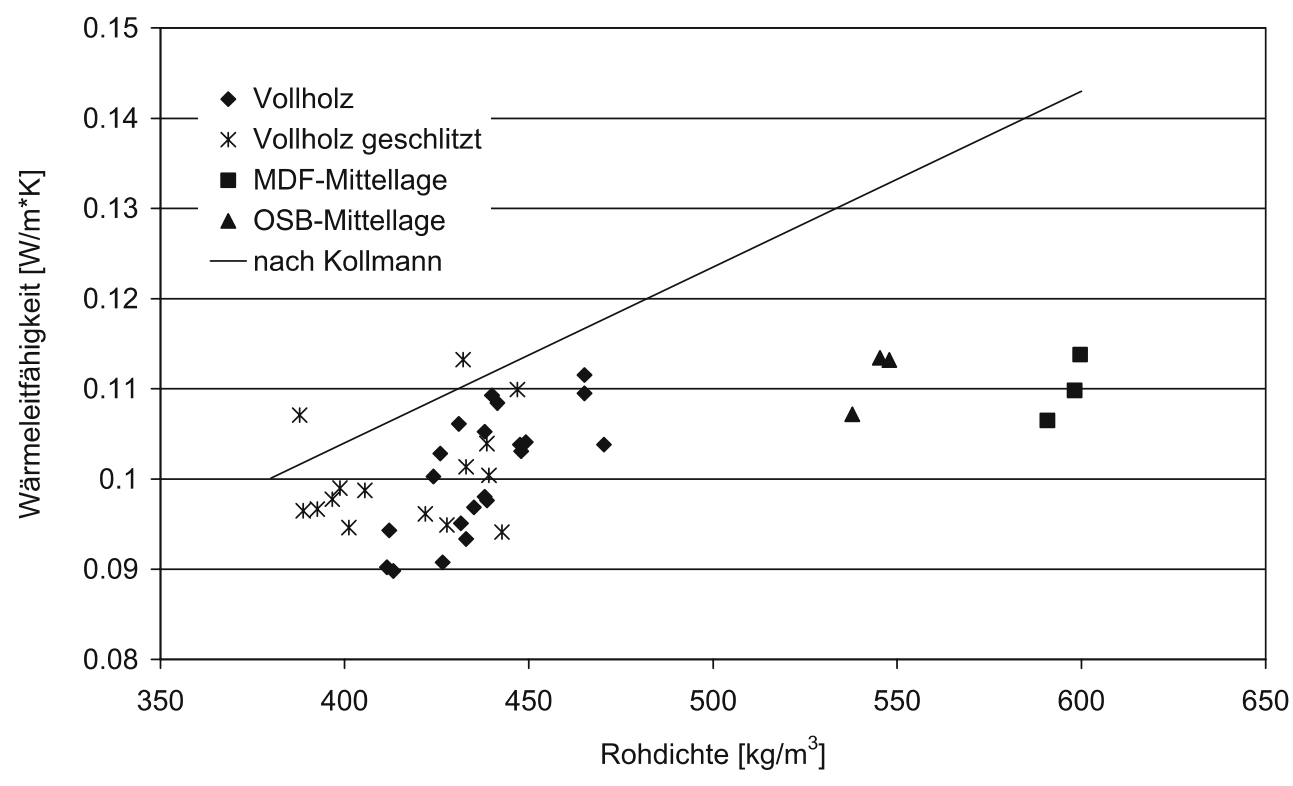

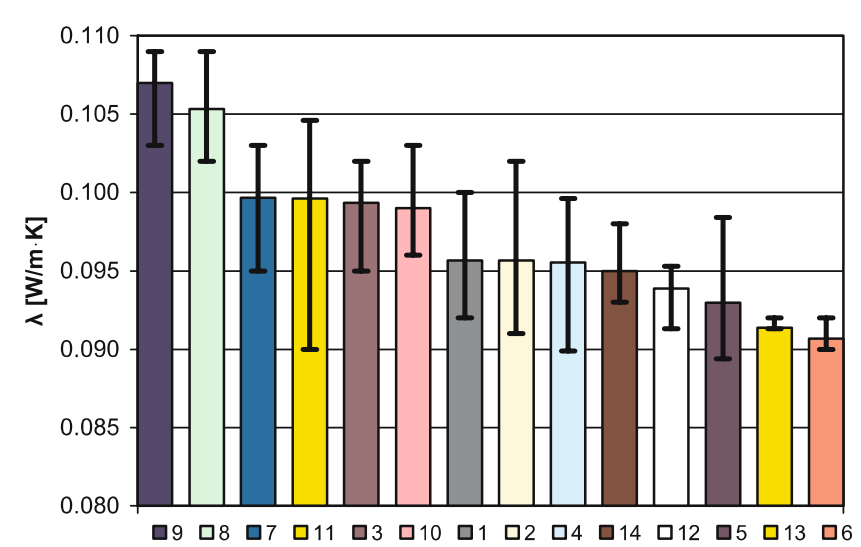

Abb. 4 Wärmeleitfähigkeit, korrigiert um die Differenzen in der Holzfeuchte und der Rohdichte (vgl. Tabelle 2)

Fig. 4 Thermal conductivity, corrected by the differences of moisture content and density (cf. Table 2)

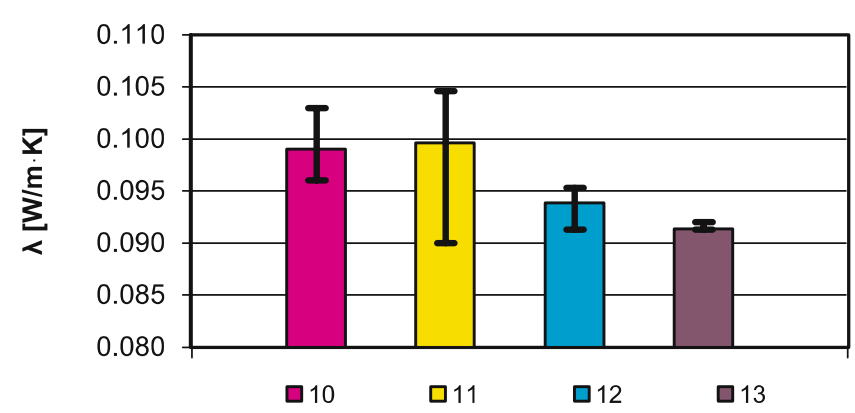

Abb.5 Wärmeleitfähigkeit als Funktion der Struktur der Mittellage (Abstand zwischen den Lamellen in der Mittellage; Varianten: 10 (10/10 $\left./ 10), 11\left(10 / 10_{5} / 10\right), 12\left(10 / 10_{10} / 10\right), 13\left(10 / 10_{30} / 10\right)\right)$

Fig. 5 Thermal conductivity as a function of the structure of the middle layer (space between the lamellas; types: $10\left(10 / 10_{0} / 10\right), 11$ $\left.\left(10 / 10_{5} / 10\right), 12\left(10 / 10_{10} / 10\right), 13\left(10 / 10_{30} / 10\right)\right)$ dungen zur Wärmeleitfähigkeit und zum Wasserdampfdiffusionswiderstand dagegen die Einzelwerte (Abb. 3, 8 und 9) oder Mittelwert sowie Maximal- und Minimalwert (Abb. 4, 5, 6 und 10).

\subsection{Wärmeleitfähigkeit}

Tabelle 2 sowie die Abbildungen 3 bis 6 zeigen eine Zusammenstellung der Messergebnisse. Die Wärmeleitfähigkeit nimmt mit zunehmender Temperatur um 0,00022-0,00048 $\mathrm{W} / \mathrm{m} \cdot \mathrm{K}^{2} \mathrm{zu}$ (Tabelle 2). Dabei ist die Zunahme bei den dicksten Platten (Varianten 3 und 5) am größten. Jedoch zeigt sich auch eine erhöhte Zunahme bei den Platten mit Hohlräumen in den Mittellagen (deutlich erkennbar bei Variante 13). Die Kennwerte der Wärmeleitfähigkeit bei $10^{\circ} \mathrm{C}$ liegen zwischen $0,088-0,107 \mathrm{~W} / \mathrm{m} \cdot \mathrm{K}$ und dadurch leicht

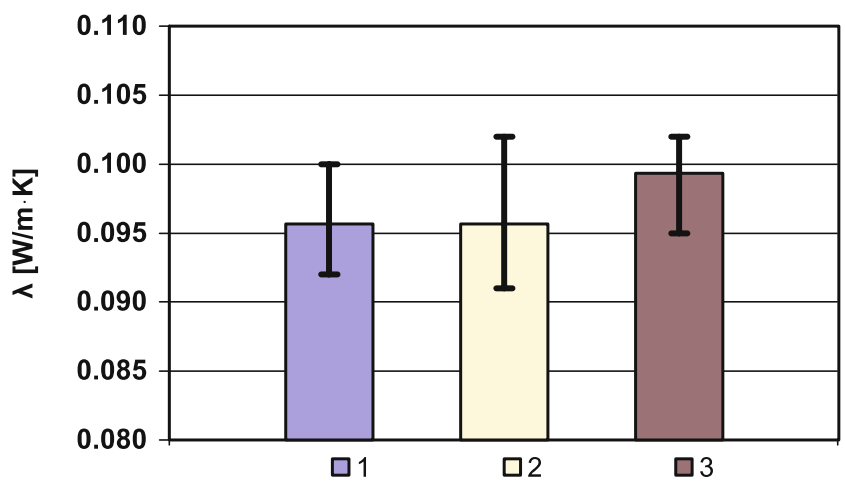

Abb. 6 Einfluss der Dicke der Mittellage auf die Wärmeleitfähigkeit (Varianten: 1 (10/10/10), 2 (10/20/10), 3 (10/40/10))

Fig. 6 Influence of the thickness of the middle layer on the thermal conductivity (types: $1(10 / 10 / 10), 2(10 / 20 / 10), 3$ (10/40/10)) 
unter den in den meisten Firmenangaben angegebenen Bereichen (meist wird ein Wert für $\lambda$ von $0,12-0,14 \mathrm{~W} / \mathrm{m} \cdot \mathrm{K}$ angegeben). Abbildung 3 stellt die Wärmeleitfähigkeit der gemessenen Platten bei $25^{\circ} \mathrm{C}$ in Abhängigkeit von der Rohdichte dar. Dabei ergeben sich fast durchwegs niedrigere Werte gegenüber der von Kollmann (1951) allgemein für Holz quer zur Faserrichtung bei $27^{\circ} \mathrm{C}$ bestimmten Wärmeleitfähigkeit (Gl. 2). Dies gilt insbesondere auch für die Platten mit einer OSB- bzw. MDF-Mittellage, welche im Verhältnis zu ihrer hohen Rohdichte sehr tiefe Wärmeleitfähigkeitswerte aufweisen.

Um die Ergebnisse der Messungen an den Platten aus reinen Holzlagen besser vergleichen zu können, wurden diese auf eine einheitliche Rohdichte und eine einheitliche Holzfeuchte umgerechnet. Es waren aber vergleichsweise geringe Differenzen vorhanden. Abbildung 4 gibt einen Überblick über die Mittel- sowie Maximal- und Minimalwerte der Wärmeleitfähigkeit aller Platten (bei den Platten aus reinen Holzlagen sind die Werte auf eine einheitliche Feuchte und Dichte korrigiert (vgl. Tabelle 2)).

Der Dichteeinfluss macht sich erwartungsgemäß auch bei den Platten mit Hohlräumen in der Mittellage bemerkbar. Je größer der Abstand zwischen den je $7 \mathrm{~cm}$ breiten Brettlamellen in der Mittellage ist, d. h. je geringer auch die Dichte der Platten ist, desto geringer ist die Wärmeleitfähigkeit (Abb. 5). Zwischen den Lamellen ohne Abstand ist dabei eine Differenz von verklebten und unverklebten Lamellen erkennbar (Varianten 1 und 10), die näher untersucht werden sollte. In Abb. 5 sind daher nur die unverklebten Varianten dargestellt. Anderseits führt die OSB bzw. MDF in der Mittellage infolge von deren deutlich größerer Rohdichte zu einem Anstieg der Wärmeleitfähigkeit (Abb. 4, Varianten 8 und 9). Ein gewisser Einfluss ist auch hinsichtlich der Orientierung der Lagen vorhanden, da die Wärmeleitfähigkeit bei Vollholz radial leicht höher ist als tangential (Niemz 1993). Diese Tendenz zeigt sich bei den Varianten 6 (tangential) und 14 (radial), jedoch nicht bei Variante 7 (tangential), da hier weitere Einflüsse wie unterschiedliche Dicke und schmälere Lamellen (höherer Klebstoffanteil) die Vergleichbarkeit erschweren. Die Dicke der Lagen wirkt sich vergleichsweise wenig aus (Abb. 6). Bei extrem dicken Mittellagen kommt es durch die vorhandene Jahrringneigung zu einem gewissen Einfluss des Winkels zwischen radialer und tangentialer Richtung.

\subsection{Gleichgewichtsfeuchte und Quellung}

Tabelle 3 zeigt die Gleichgewichtsfeuchte der Proben als Funktion der relativen Luftfeuchtigkeit. Zum Vergleich ist Fichtenvollholz eingetragen. Im unteren Bereich (bis 35\% rel. Luftfeuchte) ist die Gleichgewichtsfeuchte bei Massivholzplatten etwas höher, oberhalb von $35 \%$ niedriger als bei Vollholz. Diese Tendenz wurde auch von Pop- per et al. (2004) bestimmt. Die Ursache hierfür ist noch nicht eindeutig geklärt, hierzu laufen weitere Arbeiten. Da die Platten bei Raumtemperatur verpresst wurden, kommt die Presstemperatur nicht als Ursache in Frage. Bei Materialkombinationen Vollholz/Partikelwerkstoffe dürfte die niedrigere Gleichgewichtsfeuchte des Verbundsystems durch die geringere Gleichgewichtsfeuchte der Partikelwerkstoffe hervorgerufen werden. Die Platten mit MDFund OSB-Mittellagen haben bekanntermaßen eine etwas geringere Gleichgewichtsfeuchte als die mit VollholzMittellagen.

Tabelle 4 zeigt die differenzielle Quellung (q) der Varianten. Dabei kommt es bei allen Platten durch die kreuzweise Verleimung der Lagen zu einem deutlichen Absperreffekt bezüglich der Quellung quer zur Faser in Plattenebene. Die Varianten mit Abständen zwischen den Mittellagen (Varianten 11-13) wurden senkrecht zur Orientierung der Decklagen nicht gemessen, da der Fugenabstand teilweise die Probenbreite überschritt (Variante 13). Die Quellung parallel zur Faserrichtung der Decklagen liegt mit einem mittleren $q$ von $0,010 \% / \%$ bis $0,018 \% / \%$ etwas über der von Vollholz ( $q=0,01 \% / \%)$, was auf den Quelldruck der Mittellagen zurückzuführen ist. Quer zur Faserrichtung verhält sich die Längenquellung bei den meisten Platten ähnlich, was auf den Quelldruck der Decklagen zurückzuführen ist. Deutlich höher liegt sie jedoch bei Verwendung von OSB und MDF als Mittellage. Sie liegt etwas oberhalb der freien Quellung von MDF und OSB in Plattenebene (nach Sonderegger und Niemz (2006): $q=0,040 \% / \%$ für MDF; $q=0,028 \% / \%$ für OSB). Ebenso zeigt sich bei den Platten mit Deckla-

Tabelle 4 Differenzielle Längenquellung $q$ mit Standardabweichung zwischen $35 \%$ und $80 \%$ rel. Luftfeuchte. Parallel = parallel zum Faserverlauf der Deckschicht (Messung in der Deckschicht); quer = quer zum Faserverlauf der Deckschicht (Messung in der Mittelschicht).

Table 4 Differential in-plane swelling $q$ with standard deviation at a relative humidity of between $35 \%$ and $80 \%$. Parallel $=$ parallel to the fibre orientation of the upper layer (measured on the upper layer); quer $=$ perpendicular to the fibre orientation of the upper layer (measured on the middle layer).

\begin{tabular}{ccccc}
\hline Variante & $\begin{array}{c}\text { Plattenaufbau } \\
{[\mathrm{mm}]}\end{array}$ & $\begin{array}{c}\text { Rohdichte } \\
{\left[\mathrm{kg} / \mathrm{m}^{3}\right]}\end{array}$ & $\begin{array}{c}q \text { parallel } \\
{[\% / \%]}\end{array}$ & $\begin{array}{c}q \text { quer } \\
{[\% / \%]}\end{array}$ \\
\hline 1 & $10 / 10 / 10$ & 404 & $0,012 \pm 0,003$ & $0,016 \pm 0,003$ \\
2 & $10 / 20 / 10$ & 434 & $0,018 \pm 0,009$ & $0,011 \pm 0,003$ \\
3 & $10 / 40 / 10$ & 422 & $0,014 \pm 0,004$ & $0,010 \pm 0,004$ \\
4 & $7 / 14_{G} / 7$ & 438 & $0,010 \pm 0,007$ & $0,009 \pm 0,004$ \\
5 & $20 / 30_{G} / 20$ & 398 & $0,013 \pm 0,004$ & $0,015 \pm 0,006$ \\
6 & $10_{s t} / 10 / 10_{s t}$ & 424 & $0,015 \pm 0,005$ & $0,016 \pm 0,004$ \\
7 & $14_{s t} / 14_{s t} / 14_{s t}$ & 478 & $0,011 \pm 0,003$ & $0,020 \pm 0,011$ \\
8 & $10 / 19_{M D F} / 10$ & 571 & $0,014 \pm 0,006$ & $0,046 \pm 0,004$ \\
9 & $10 / 17_{O S B} / 10$ & 536 & $0,011 \pm 0,004$ & $0,031 \pm 0,005$ \\
10 & $10 / 10_{0} / 10$ & 445 & $0,018 \pm 0,011$ & $0,017 \pm 0,005$ \\
11 & $10 / 10_{5} / 10$ & 430 & $0,014 \pm 0,007$ & - \\
12 & $10 / 10_{10} / 10$ & 419 & $0,012 \pm 0,005$ & - \\
13 & $10 / 10_{30} / 10$ & 393 & $0,015 \pm 0,007$ & - \\
14 & $10_{l} / 10 / 10_{l}$ & 438 & $0,010 \pm 0,004$ & $0,024 \pm 0,013$ \\
\hline
\end{tabular}


gen mit liegenden Jahrringen (Variante 14) eine deutlich stärkere Quellung, bedingt durch die tangentiale Ausrichtung der Decklagen.

\subsection{Wasserdampfdiffusionswiderstand}

Tabelle 5 und Abb. 8-10 zeigen die Versuchsergebnisse. Deutlich ist der unter Verwendung aller Platten aus Vollholz berechnete Einfluss der Dichte auf den Wasserdampfdiffusionswiderstand erkennbar (Abb. 8). Auch der Unterschied zwischen liegend und stehend orientierten Decklagen ist gut erkennbar (Abb. 9). Bei liegenden Jahrringen macht sich die in radialer Richtung geringere Wasserdampfdiffu-

Tabelle 5 Wasserdampfdiffusionswiderstandszahl $\mu$, Luftschichtdicke $\mathrm{s}_{d}$ und Holzfeuchte (mittlere Holzfeuchte im Ausgleichszustand)

Table 5 Water vapour resistance factor $\mu$, water vapour diffusionequivalent air layer thickness $\mathrm{s}_{d}$ and moisture content (mean moisture content at the equilibrium)

\begin{tabular}{lcrrr}
\hline Variante & Plattenaufbau & $\mu[-]$ & $s_{d}[\mathrm{~m}]$ & $\begin{array}{c}\text { Holzfeuchte } \\
{[\%]}\end{array}$ \\
\hline 1 & $10 / 10 / 10$ & 134,2 & 3,77 & 9,2 \\
2 & $10 / 20 / 10$ & 122,4 & 4,85 & 10,0 \\
3 & $10 / 40 / 10$ & 148,3 & 8,75 & 10,8 \\
4 & $7 / 14_{G} / 7$ & 109,2 & 2,89 & 9,5 \\
5 & $20 / 30_{G} / 20$ & 95,7 & 6,64 & 10,8 \\
6 & $10_{s t} / 10 / 10_{s t}$ & 120,7 & 3,43 & 9,2 \\
7 & $14_{s t} / 14_{s t} / 14_{s t}$ & 165,7 & 7,08 & 9,4 \\
8 & $10 / 19_{M D F} / 10$ & 109,0 & 4,17 & 7,6 \\
9 & $10 / 17_{O S B} / 10$ & 159,1 & 5,94 & 8,3 \\
10 & $10 / 10_{0} / 10$ & 124,9 & 3,62 & 9,3 \\
11 & $10 / 10_{5} / 10$ & 117,6 & 3,40 & 9,5 \\
12 & $10 / 10_{10} / 10$ & 81,1 & 2,35 & 9,7 \\
13 & $10 / 10_{30} / 10$ & 69,8 & 2,02 & 9,6 \\
14 & $10_{l} / 10 / 10_{l}$ & 91,5 & 2,72 & 9,0 \\
\hline
\end{tabular}

sionswiderstandszahl im Vergleich zu stehenden Jahrringen bemerkbar (Diffusion in tangentialer Richtung). Dies deckt sich mit Arbeiten von Vanek und Teischinger (1989) an Vollholz (Abb. 7). Das Schlitzen der Mittellage und die Verwendung von Hohlräumen zwischen den Brettern der Mittellage führen zu einer deutlichen Reduzierung des Wasserdampfdiffusionswiderstandes (Abb. 10). Auch der im Vergleich zum Vollholz unterschiedliche Wasserdampfdiffusionswiderstand von MDF und OSB ist in einer niedrigeren (Massivholzplatte mit MDF-Mittellage) bzw. erhöhten Wasserdampfdiffusionswiderstandszahl (Massivholzplatte mit OSB-Mittellage) erkennbar (Tabelle 5, Varianten 8 und 9). Insgesamt ist eine recht große Streuung der Messwerte vorhanden.

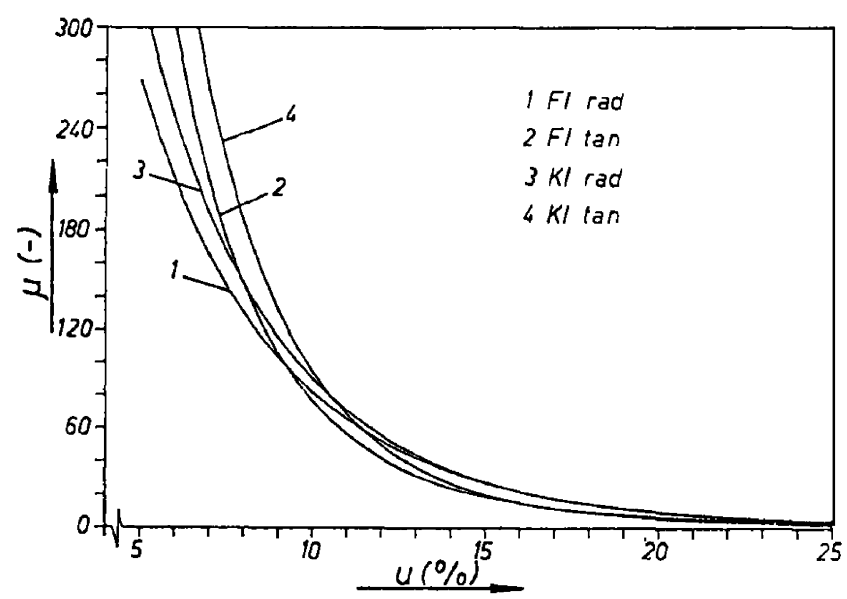

Abb. 7 Einfluss der Holzfeuchte und der Schnittrichtung auf den Wasserdampfdiffusionswiderstand nach Vanek und Teischinger (1989) Fig. 7 Influence of the moisture content and the cut direction on the water vapour resistance according to Vanek und Teischinger (1989)
Abb. 8 Wasserdampfdiffusionswiderstand in Abhängigkeit von der Dichte unter Berücksichtigung der Varianten: $1,2,3,6,7$ und 10

Fig. 8 Water vapour resistance depending on the density for the types: $1,2,3,6,7$ and 10

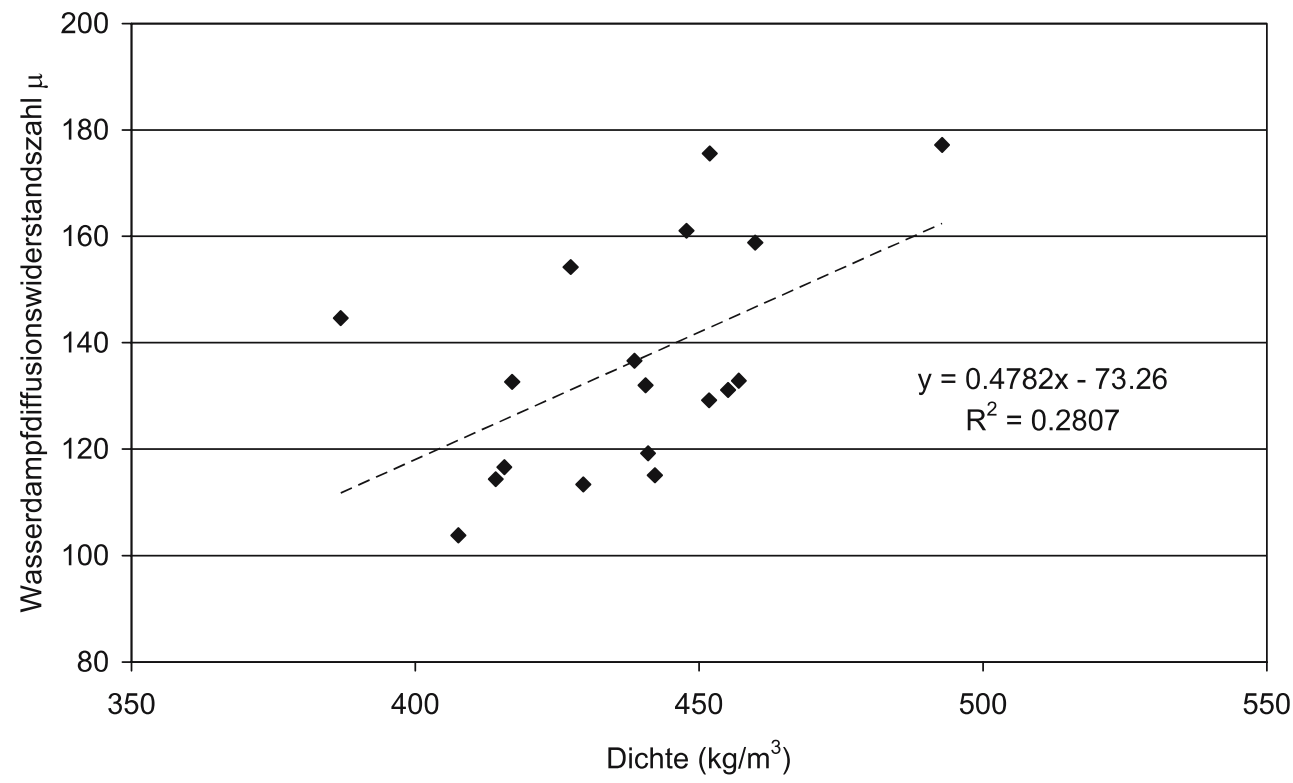




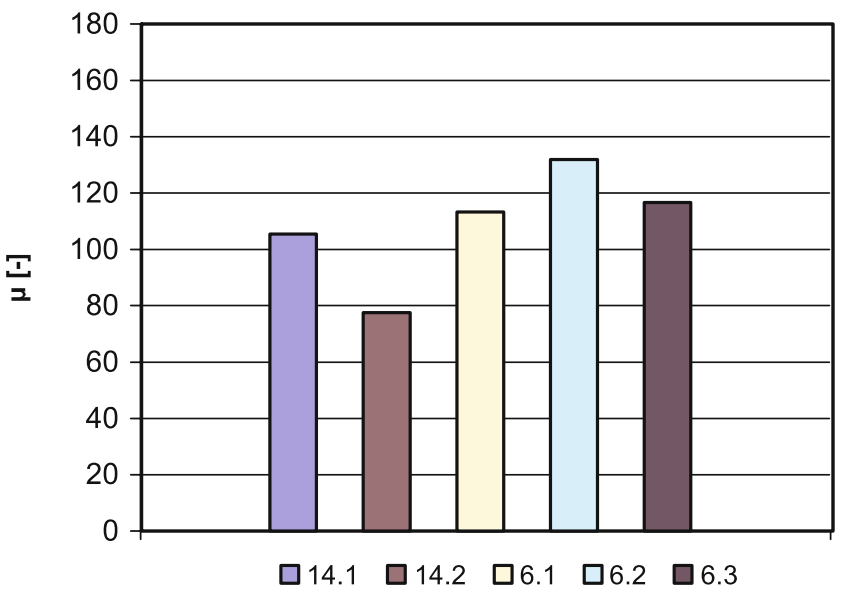

Abb. 9 Einfluss der Schnittrichtung in den Decklagen (Einzelwerte der Varianten) auf den Wasserdampfdiffusionswiderstand (Varianten: $\left.6\left(10_{\text {st }} / 10 / 10_{\text {st }}\right), 14\left(10_{l} / 10 / 10_{l}\right)\right)$

Fig. 9 Influence of the cut direction on the water vapour resistance (types: $\left.6\left(10_{\mathrm{st}} / 10 / 10_{\mathrm{st}}\right), 14\left(10_{l} / 10 / 10_{l}\right)\right)$

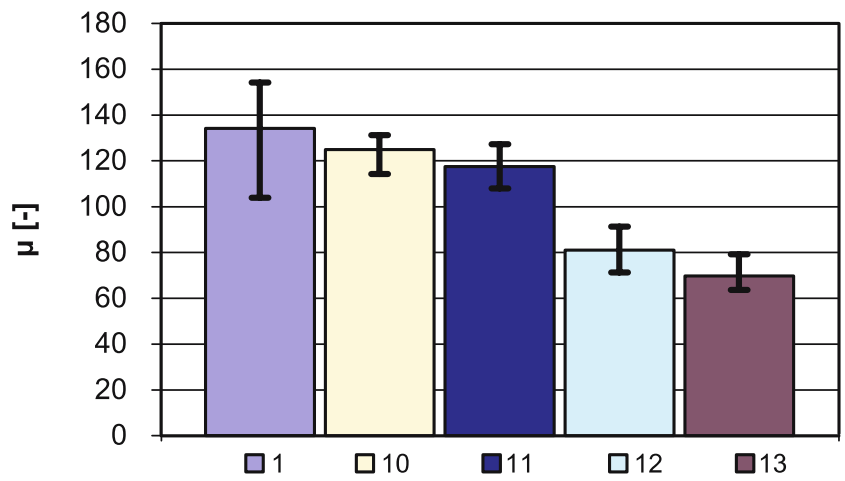

Abb. 10 Einfluss des Abstandes zwischen den Lamellen in der Mittellage auf den Wasserdampfdiffusionswiderstand (Varianten: 1 $(10 / 10 / 10), 10\left(10 / 10_{0} / 10\right), 11\left(10 / 10_{5} / 10\right), 12\left(10 / 10_{10} / 10\right), 13$ $\left.\left(10 / 10_{30} / 10\right)\right)$

Fig. 10 Influence of the space between the lamellas in the middle layer on the water vapour resistance (types: $1(10 / 10 / 10), 10$ $\left.\left(10 / 10_{0} / 10\right), 11\left(10 / 10_{5} / 10\right), 12\left(10 / 10_{10} / 10\right), 13\left(10 / 10_{30} / 10\right)\right)$

\section{Zusammenfassung und Schlussfolgerungen}

An labortechnisch hergestellten Massivholzplatten wurden Wärmeleitfähigkeit, Gleichgewichtsfeuchte, Längenquellung und Wasserdampfdiffusionswiderstand bestimmt. Die Wärmeleitfähigkeit der im Normalklima klimatisierten Massivholzplatten liegt im Bereich von $0,09-0,11 \mathrm{~W} / \mathrm{m} \cdot \mathrm{K}$. Die Wärmeleitfähigkeit korreliert mit der Rohdichte. Hohlräume in der Mittellage (Schlitze, Abstände zwischen den einzelnen Brettlamellen) führen tendenziell mit zunehmendem Lamellenabstand zu einer geringen Abnahme der Wärmeleitfähigkeit. Werden die Decklagen mit liegenden Jahrringen angeordnet, ist die Wärmeleitfähigkeit leicht höher als mit stehenden. Unter Berücksichtigung der Streuung der Werte sind die Unterschiede jedoch ge- ring. Auf den Wasserdampfdiffusionswiderstand wirken sich die Orientierung der Decklagen (in radialer Richtung niedrigerer Wasserdampfdiffusionswiderstand als in tangentialer Richtung) und Hohlräume in der Mittellage (Abfall des Wasserdampfdiffusionswiderstandes mit $\mathrm{Zu}$ nahme der Hohlräume) aus. Der Einfluss von Hohlräumen in den Mittellagen ist jedoch deutlich größer als derjenige der Orientierung der Decklagen. Die Gleichgewichtsfeuchte liegt oberhalb von $35 \%$ rel. Luftfeuchte etwas unterhalb derjenigen von Fichtenholz. Bei Holzwerkstoffmittellagen ist sie durch die geringere Ausgleichsfeuchte der Holzpartikelwerkstoffe geringer als bei reinen Vollholzmittellagen.

\section{Literatur}

Blaß HJ, Fellmoser P (2003) Bemessung von Mehrschichtplatten. Bauen Holz 8:36-39

DIN EN ISO 12572 (2001) Wärme- und feuchtetechnisches Verhalten von Baustoffen und Bauprodukten - Bestimmung der Wasserdampfdurchlässigkeit. Beuth Verlag $\mathrm{GmbH}$, Berlin, 26 Seiten

DIN 52183 (1977) Prüfung von Holz - Bestimmung des Feuchtigkeitsgehaltes. Beuth Verlag GmbH, Berlin, 2 Seiten

DIN 52184 (1979) Prüfung von Holz - Bestimmung der Quellung und Schwindung. Beuth Verlag GmbH, Berlin, 4 Seiten

DIN 52612 Teil 1 (1979) Wärmeschutztechnische Prüfungen Bestimmung der Wärmeleitfähigkeit mit dem Plattengerät. Durchführung und Auswertung. Beuth Verlag GmbH, Berlin, 6 Seiten

DIN 68705-5 Beiblatt 1 (1980) Bau-Furniersperrholz aus Buche; Zusammenhänge zwischen Plattenaufbau, elastischen Eigenschaften und Festigkeiten. Beuth Verlag GmbH, Berlin, 6 Seiten

Donzé M, Niemz P (2004) Orientierende Untersuchungen zur Modellierung der Eigenschaften dreischichtiger Massivholzplatten. HOLZ (1):27-31

Kreuzinger H (1991) Platten, Scheiben, Schalen. Bauen Holz 1: 34-39

Kollmann F (1951) Technologie des Holzes und der Holzwerkstoffe. Erster Band: Anatomie und Pathologie, Chemie, Physik, Elastizität und Festigkeit. 2. Auflage. Springer, Berlin Göttingen Heidelberg, 1050 Seiten

Krug D (1999) Der Holzwerkstoff Massivholzplatte. Ausgewählte Material- und Gebrauchseigenschaften dreilagiger Massivholzplatten aus Nadelholz für konstruktive Zwecke. Tagungsband, 3. IHD Kolloquium, Dresden, 09.12.1999

Merz K, Fischer J, Brunner R, Baumberger M (1997) Holzprodukte für den statischen Einsatz. Teil 1: Plattenförmige Holzprodukte. Lignatec, Zürich, 28 Seiten

Niemz P (1993) Physik des Holzes und der Holzwerkstoffe. DRWVerlag, Leinfelden-Echterdingen, 243 Seiten

Popper R, Niemz P, Eberle G (2004) Untersuchungen zur Gleichgewichtsfeuchte und Quellung von Massivholzplatten. Holz RohWerkst 62(3):209-217

Radovic B, Cheret P, Heim F (2001) Informationsdienst Holz: Holzbau Handbuch. Reihe 4: Baustoffe. Teil 4: Holzwerkstoffe. Folge 1: Konstruktive Holzwerkstoffe. 2. Auflage. Arbeitsgemeinschaft Holz e.V., Düsseldorf, 26 Seiten

Tobisch S, Krug D (2002a) Dreilagige Massivholzplatten mit substituierter Mittellage. HOLZ 3:41-45 
Tobisch S, Krug D (2002b) Die neue Mitte. DDS - Das Magazin für Möbel und Ausbau 6:40-43

Tobisch S, Krug D (2002c) Neuartige mehrschichtige Verbundplatten. Mobil Oil Symposium, Bremen, 12.-14.9.2002

Sonderegger W, Niemz P (2006) Untersuchungen zur Quellung und Wärmedehnung von Faser-, Span- und Sperrholzplatten. Holz Roh- Werkst 64(1):11-20
Steiger B, Niemz P (2004) Untersuchungen zu ausgewählten Einflussfaktoren auf die Eigenschaften von dreischichtigen Massivholzplatten. HOLZ 1:29-32

Vanek M, Teischinger A (1989) Diffusionskoeffizienten und Diffusionswiderstandszahlen von verschiedenen Holzarten. Holzforsch Holzverw 41(1):3-6 